\title{
The Banff International Research Station for Mathematical Innovation and Discovery \\ NassifGhoussoub
}

The Banff International Research Station (BIRS) was established in 2003 to address the imperatives of collaborative research and cross-disciplinary synergy. It provides an environment for creative, intense, and prolonged interactions between mathematical scientists and researchers in related areas of science and engineering. BIRS is also about multiplying opportunities, providing intellectual access, facilitating collaborative problem solving, incubating new research projects, settling intellectual controversies, and disseminating new discoveries, while also providing a forum for accelerated training, networking, and job prospecting for new generations of mathematical scientists.

While many other research-enabling institutes exist around the globe, the

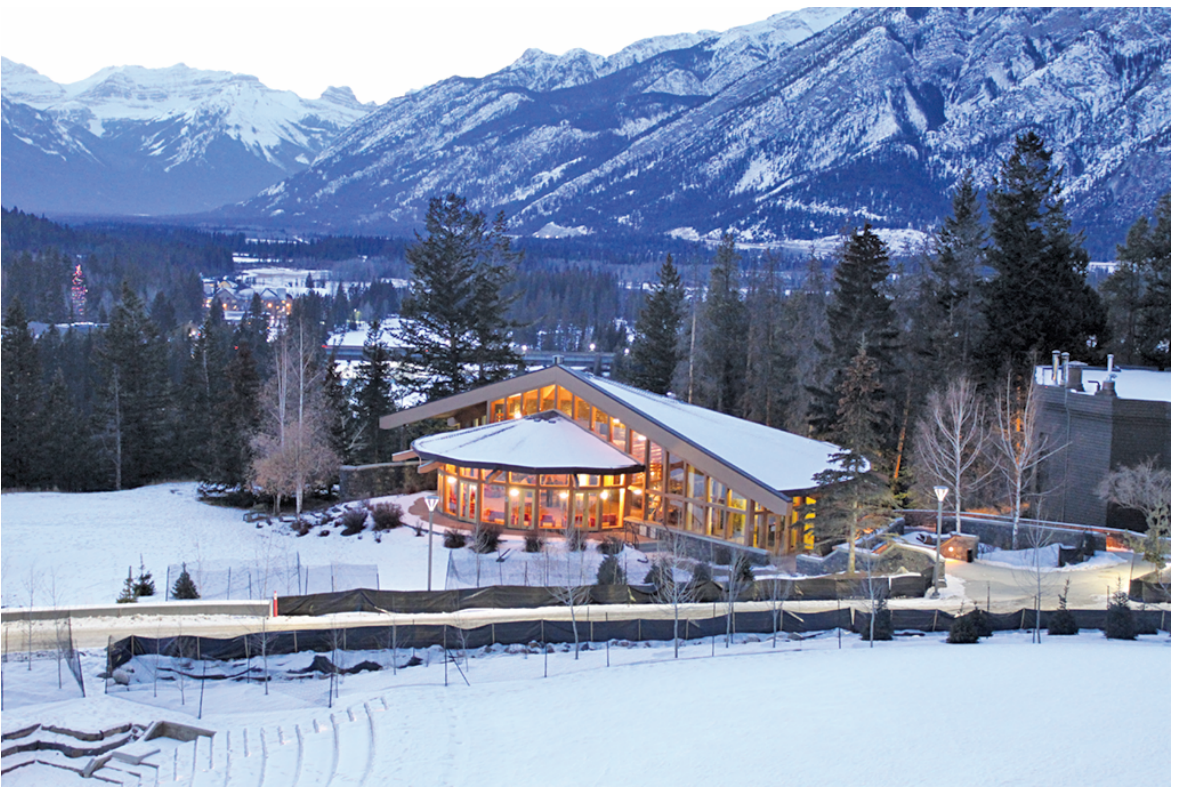
BIRS model offers several distinctive features: The Station provides a setting that is conducive to uninterrupted scientific interaction by way of focused, easily organized research workshops. Each year, its pro-

Nassif Ghoussoub is the director of the Banff International Research Station. He is a professor of mathematics and a Distinguished University Scholar at The University of British Columbia. His email address is bi rs-di rector abirs.ca.

For permission to reprint this article, please contact: reprint-permission aams.org.

DOI: http://dx.doi.org/10.1090/noti1876

grams cover a broad range of the mathematical sciences and respond quickly to new and exciting developments. Even within the category of institutions that deliver in the same format, such as the MFO (Germany), CIRM (France), and AIM (USA), BIRS distinguishes itself by being distinctly multi-disciplinary, particularly receptive to emerging-possibly risky - areas of research, and by its commitment to provide equal access to all scientists worldwide through an open and highly competitive peer-reviewed process. BIRS is also a multinational collaborative effort that illustrates 
how scientific leadership can transcend not only provincial and national, but also disciplinary boundaries in the global quest to advance scientific discovery and innovation.

BIRS embraces all aspects of quantitative and analytic research. Its programs span pure, applied, computational, and industrial mathematics; statistics; and computer science. Its workshops often involve physicists, biologists, engineers, economists, and financial mathematicians. In addition, BIRS hosts leadership retreats, student modeling camps, First Nations workshops, training sessions for math Olympiad teams, and "ateliers" in scientific writing.

The retreat-like atmosphere at BIRS is ideal for ensuring a creative environment for the exchange of ideas, knowledge, and methods within the mathematical sciences and their vast array of applications in science and engineering. An added intellectual feature of the Station is its location within the site of the world-renowned Banff Centre for Arts and Creativity in Alberta, which is already internationally recognized as a place of high culture with programs in music and sound, and the written, visual, and performing arts that draw in many hundreds of artists, scholars, and intellectual leaders from around the world.

BIRS is committed to providing intellectual access and opportunities on a large scale and in an open competitive process by making a place of cutting edge research accessible to a large number of scientists. Every year, a call for proposals is sent to the international mathematical science community as BIRS provides equal access to all researchers regardless of geographic location or scientific expertise as long as it is anchored on solid mathematical, statistical, or computational grounds. Applications are selected on a competitive basis, using the criteria of excellence and relevance, by an international scientific panel of thirty experts drawn from across the entire breadth of the mathematical sciences and related areas.

BIRS's main mode of operation is to competitively select and run weekly workshops, each devoted to a specific area of high significance while involving forty-two researchers from around the world. Each workshop has its own personality, yet they all share the common objective of encouraging an atmosphere that fosters innovative ideas and a collaborative spirit. The extraordinary response to the opportunities at BIRS leads to extremely high-quality competitions: more than 250 applications for workshops were received for the 2019 program alone. This guarantees high standards of excellence, a diverse scientific program, and a multinational collaborative effort.

BIRS also hosts teams of two to four researchers for periods of one or two weeks to allow collaborative, distraction-free research and the completion of major scientific projects. The Station's setting has also been ideal for summer schools and focused collaborative research groups. The weekends are also used for two-day workshops and university-industry interactions.
BIRS is a key resource in the global effort for promoting diversity in the sciences. In particular, it addresses in its own way the challenges of increasing participation of underrepresented groups in STEM. Every year the station hosts several exclusively "Women in X" workshops. In 2019, the $\mathrm{X}$ will stand for Geometry, Numerical Methods for PDEs \& Applications, Analysis, and Commutative Algebra. These four workshops can count on the participation of about 150 women mathematicians from all over the world. Accommodations suitable for families are available at BIRS, with special provisions for women with infants and nursing mothers. Since 2015 BIRS has provided financial support for workshop participants who are travelling with children requiring day-care services. BIRS has led the way in addressing science and education issues for aboriginal people.

In 2012, BIRS made its programs accessible to the world's scientific community in virtual space, via live video streaming, recordings, and broadcasting produced by a state-ofthe-art automated production system. About 125 new videos are recorded every month and more than 10,000 of them have already been posted on the BIRS website.

BIRS "underscores how international cooperation adds up to more than what any nation could accomplish alone." These words of the former director of the NSF, Rita Colwell, go to the heart of this project, since BIRS represents a new level of scientific cooperation in North America. Indeed, a rewarding unique aspect of BIRS is that it is a joint Canada-US-Mexico initiative, which is funded by Mexico's Consejo Nacional de Ciencia y Tecnología (CONACYT), Alberta Innovation, the US National Science Foundation (NSF), and Canada's Natural Science and Engineering Research Council (NSERC). A partnership of this scale is providing new and exciting opportunities for North American faculty and graduate students, giving them access to their international colleagues at the highest levels and across all mathematical disciplines.

This collaboration reached a new milestone in 2014, when the Government of Mexico awarded an infrastructure

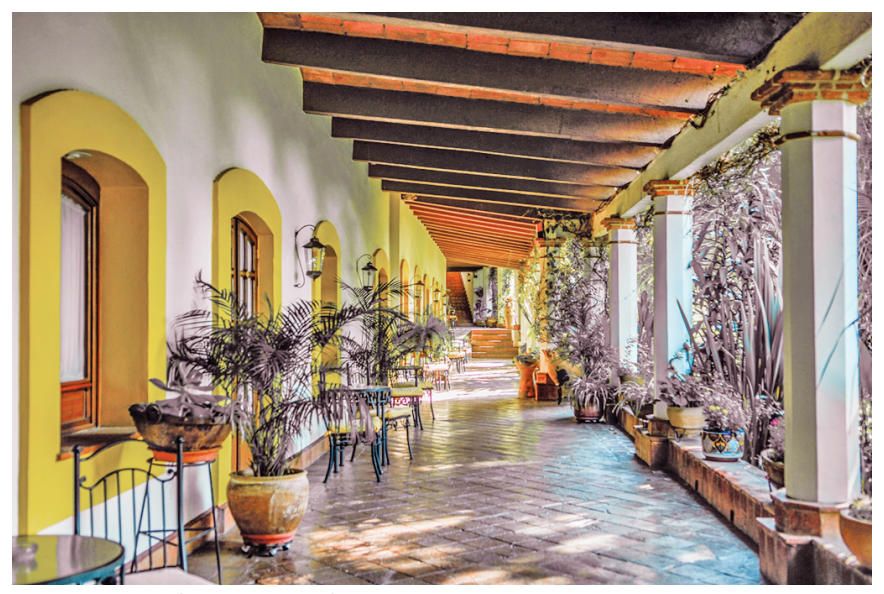

The current (temporary) CMO facilities in Oaxaca. 


\section{COMMUNICATION}

grant for the construction of an affiliated research facility in Oaxaca so that BIRS could run an additional twenty-five workshops per year. Casa Mathematica Oaxaca (CMO) was born in 2015, and the construction of a new and permanent facility there will be starting soon thanks to generous funding from CONACYT and the Universidad Nacional Autónoma de México (UNAM). Most recently, the Simons Foundation approved funding for the operating costs of several BIRS workshops at CMO. Furthermore, in a new and promising pilot program, BIRS will run ten additional workshops in 2020 jointly with the nascent Institute of Advanced Studies in Hangzhou, China. As such, the 2020 BIRS program will consist of eighty-five weekly workshops giving access to 3,500 researchers from hundreds of institutions in more than eighty countries. This groundbreaking development opens a much-needed new era for international collaborative research in the mathematical sciences.

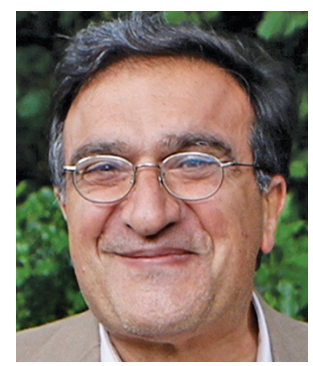

Nassif Ghoussoub

\section{Credits}

All photos are courtesy of BIRS and CMO.

\section{$\boldsymbol{G H}$ zürich}

\section{Professor of Mathematics and Physics}

$\rightarrow$ The Department of Mathematics (www.math.ethz.ch) and the Department of Physics (www.phys.ethz.ch) at ETH Zurich invites applications for the above-mentioned position. The new professor will be based in the Department of Mathematics and associated to the Department of Physics.

$\rightarrow$ Applicants should demonstrate an outstanding research record and a proven ability to direct research work of high quality. The successful candidate should have a strong background and a worldwide reputation in mathematical physics as well as excellent teaching skills. Teaching responsibilities will mainly involve undergraduate (German or English) and graduate courses (English) for students in mathematics, physics and engineering.

$\rightarrow$ Please apply online:

www.facultyaffairs.ethz.ch

$\rightarrow$ Applications should include a curriculum vitae, a list of publications, a statement of future research and teaching interests, and a description of the three most important achievements. The letter of application should be addressed to the President of ETH Zurich, Prof. Dr. Joël Mesot. The closing date for applications is 15 September 2019. ETH Zurich is an equal opportunity and family friendly employer and is responsive to the needs of dual career couples. We specifically encourage women to apply. 